\title{
Clinical Study \\ Prevalence of Dyslipidemia in Patients Receiving Health Checkups: A Hospital-Based Study
}

\author{
Kao-Chi Cheng, ${ }^{1,2}$ Yu-Lung Chen,, ${ }^{1,2}$ and Shih-Wei Lai ${ }^{1,2}$ \\ ${ }^{1}$ School of Medicine, China Medical University, Taichung 40447, Taiwan \\ ${ }^{2}$ Department of Family Medicine, China Medical University Hospital, Taichung 40447, Taiwan
}

Correspondence should be addressed to Shih-Wei Lai, wei@mail.cmuh.org.tw

Received 6 June 2011; Accepted 21 August 2011

Academic Editor: Jean Davignon

Copyright (C) 2011 Kao-Chi Cheng et al. This is an open access article distributed under the Creative Commons Attribution License, which permits unrestricted use, distribution, and reproduction in any medium, provided the original work is properly cited.

\begin{abstract}
We used the dataset from one medical center in Taiwan to explore the prevalence of dyslipidemia, which included 2695 subjects receiving private health checkups in 2003-2004. The overall prevalence of hypercholesterolemia was $53.3 \%$ in men and $48.2 \%$ in women $(P=0.008)$. The overall prevalence of hypertriglyceridemia was $29.3 \%$ in men and $13.7 \%$ in women $(P<0.001)$. The overall prevalence of elevated LDL level was $50.7 \%$ in men and $37.9 \%$ in women $(P<0.001)$. The overall prevalence of low HDL level was $47.4 \%$ in men and $53 \%$ in women $(P=0.004)$.
\end{abstract}

We analyzed 2695 subjects who received private health checkups at their own expense at one medical center located at the Taichung city in mid-Taiwan from 2003 to 2004. There were 1526 men $(56.6 \%)$ and 1169 women $(43.4 \%)$. The mean age was 49.2 years old (standard deviation 12.2 , range from 20 to 84). Hypercholesterolemia was defined as total cholesterol level $\geq 200 \mathrm{mg} / \mathrm{dL}$ [1]. Hypertriglyceridemia was defined as triglyceride level $\geq 150 \mathrm{mg} / \mathrm{dL}$ [2]. Elevated lowdensity lipoprotein cholesterol (LDL) level was defined as LDL level $\geq 130 \mathrm{mg} / \mathrm{dL}$ [2]. Low level of high-density lipoprotein cholesterol (HDL) was defined as $\mathrm{HDL}<40 \mathrm{mg} / \mathrm{dL}$ in men and $<50 \mathrm{mg} / \mathrm{dL}$ in women [2].

In Figure 1, the overall prevalence of hypercholesterolemia was $53.3 \%$ in men and $48.2 \%$ in women $(P=$ 0.008 ). In the group aged 20 to 39 , men had higher prevalence of hypercholesterolemia than women $\operatorname{did}(48.2 \%$ versus $22.6 \%, P<0.001)$. In the group aged 65 and over, women had higher prevalence than men did $(63.5 \%$ versus $47.2 \%$, $P=0.003)$. There was no significant difference between men and women in the group aged 40 to 64 (55.9\% versus 53.8\%, $P=0.368)$. The prevalence of hypercholesterolemia increased with age in women $(P<0.001)$.

In Figure 2, the overall prevalence of hypertriglyceridemia was $29.3 \%$ in men and $13.7 \%$ in women $(P<0.001)$.
Men had higher prevalence of hypertriglyceridemia than women did in the group aged 20 to 39 and in the group aged 40 to $64(P<0.001$ and $P<0.001$, resp.). There was no significant difference between men and women in the group aged 65 and over (18.2\% versus $26.4 \%, P=0.077$ ). The prevalence of hypertriglyceridemia increased with age in women $(P<0.001)$.

In Figure 3, the overall prevalence of elevated LDL level was $50.7 \%$ in men and $37.9 \%$ in women $(P<0.001)$. Men had higher prevalence of elevated LDL level than women did in the group aged 20 to 39 and in the group aged 40 to 64 ( $P<0.001$ and $P<0.001$, resp.). There was no significant difference between men and women in the group aged 65 and over $(46.0 \%$ versus $48.6 \%, P=0.637)$. The prevalence of elevated LDL level increased with age in women $(P<0.001)$.

In Figure 4, the overall prevalence of low HDL level was $47.4 \%$ in men and $53 \%$ in women $(P=0.004)$. Women had higher prevalence of low HDL level than men did in the group aged 40 to 64 and in the group aged 65 and over ( $P=0.047$ and $P=0.001$, resp.). There was no significant difference between men and women in the group aged 20 to $39(46.3 \%$ versus $47.1 \%, P=0.857)$. The prevalence of low HDL level increased with age in women $(P=0.014)$. 


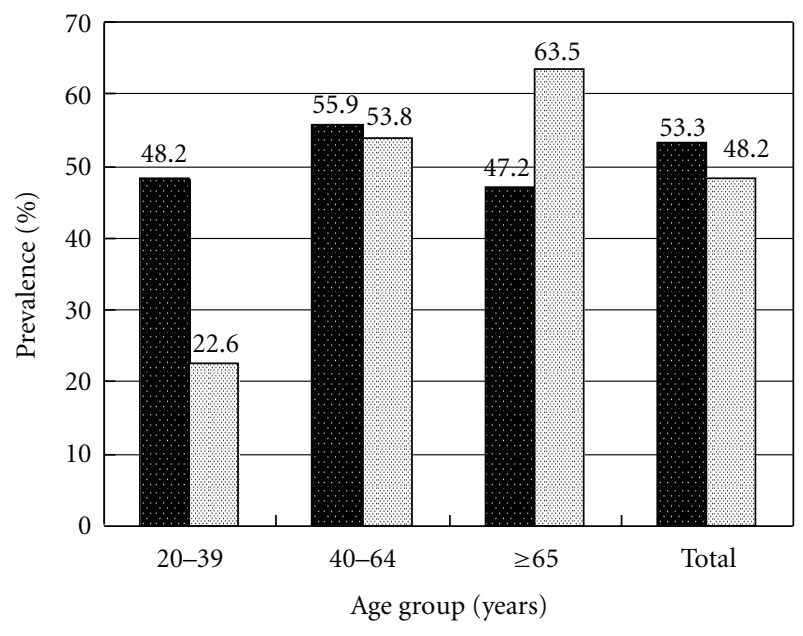

Men

Women

Figure 1: Prevalence of hypercholesterolemia in gender and three age groups.

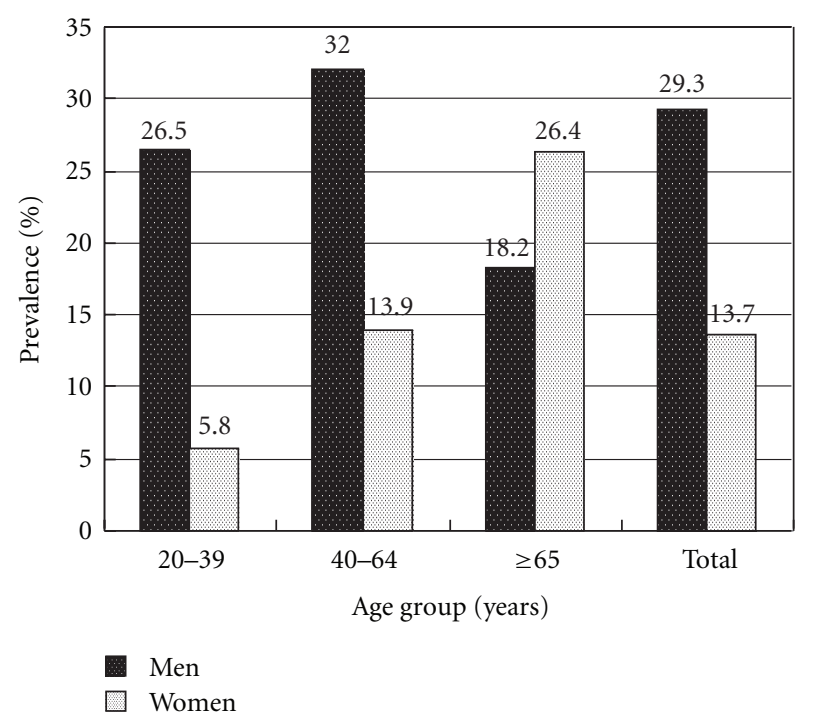

FIgURe 2: Prevalence of hypertriglyceridemia in gender and three age groups.

In 2010, cardiovascular disease and cerebrovascular disease are the second and the third leading causes of death in Taiwan [3]. Previous reports have suggested dyslipidemia, including hypercholesterolemia (elevated total cholesterol level), hypertriglyceridemia, elevated LDL level, and low HDL level, is a major risk factor for cardiovascular disease and ischemic stroke [4-6]. In this present study, the overall prevalence of dyslipidemia is relatively high, including hypercholesterolemia 51.1\%, hypertriglyceridemia $22.5 \%$, elevated LDL level $45.1 \%$, and low HDL level $49.8 \%$. In addition, the prevalence rates of hypercholesterolemia, hypertriglyceridemia, elevated LDL level, and low HDL level significantly increase with age in women. That is, the prevalence of dyslipidemia in women is lower in the group

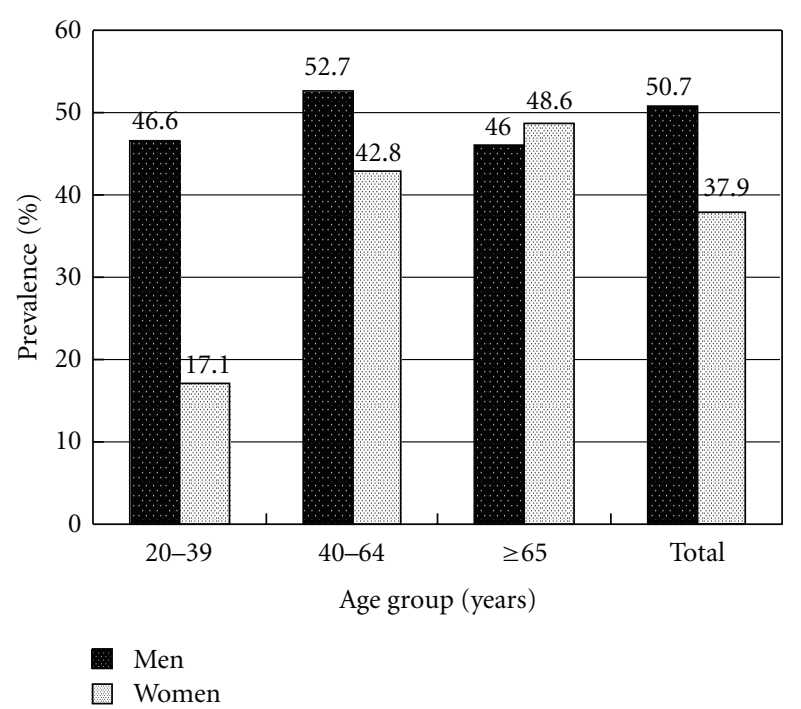

Figure 3: Prevalence of elevated LDL level in gender and three age groups.

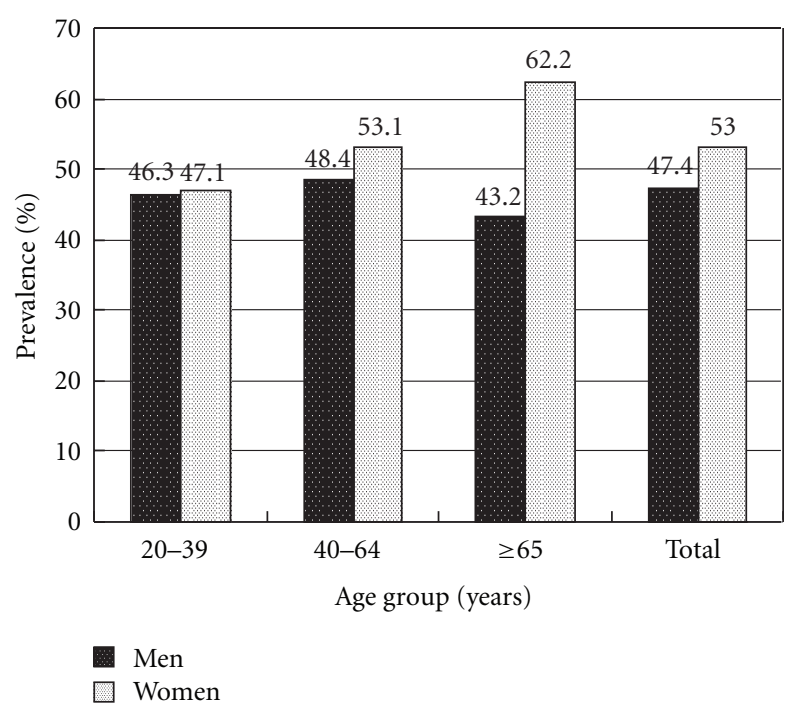

Figure 4: Prevalence of low HDL level in gender and three age groups.

aged 20-39 than in the group aged 40 and over, but no similar trend is found in men. This result further reveals that the status of all serum lipids in women aged 20-39 is significantly better than the other age groups. However, in this dataset, there was only blood data, no mention whether these patients were using lipid-lowering drugs. Thus, the prevalence of dyslipidemia might be underestimated. Meanwhile, we hope these findings are to guide public health interventions to manage dyslipidemia issue in Taiwan.

\section{Acknowledgment}

Kao-Chi Cheng and Yu-Lung Chen contributed equally to this study. 


\section{References}

[1] "Third report of the National Cholesterol Education Program (NCEP) expert panel on detection, evaluation, and treatment of high blood cholesterol in adults (adult treatment panel III) final report," Circulation, vol. 106, pp. 3143-3421, 2002.

[2] S. M. Grundy, J. I. Cleeman, S. R. Daniels et al., "Diagnosis and management of the metabolic syndrome: an American Heart Association/National Heart, Lung, and Blood Institute scientific statement," Circulation, vol. 112, no. 17, pp. 2735-2752, 2005.

[3] "Department of Health, Taiwan: main causes of death in 2010," 2011, http://www.doh.gov.tw/ufile/doc/99\%e6\%ad\%bb\%e5\% 9b\%a0\%e7\%b0\%a1\%e5\%a0\%b1(\%e5\%88\%97\%e5\%8d\%b0 \%e7\%89\%88)1000615.pdf.

[4] G. Assmann, H. Schulte, and A. von Eckardstein, "Hypertriglyceridemia and elevated lipoprotein(a) are risk factors for major coronary events in middle-aged men," American Journal of Cardiology, vol. 77, no. 14, pp. 1179-1184, 1996.

[5] K. Tziomalos, V. G. Athyros, A. Karagiannis, and D. P. Mikhailidis, "Dyslipidemia as a risk factor for ischemic stroke," Current Topics in Medicinal Chemistry, vol. 9, no. 14, pp. 12911297, 2009.

[6] A. Patel, "Cholesterol, coronary heart disease, and stroke in the Asia Pacific region," International Journal of Epidemiology, vol. 32, no. 4, pp. 563-572, 2003. 


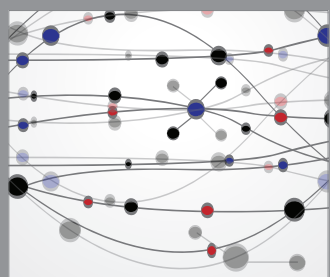

The Scientific World Journal
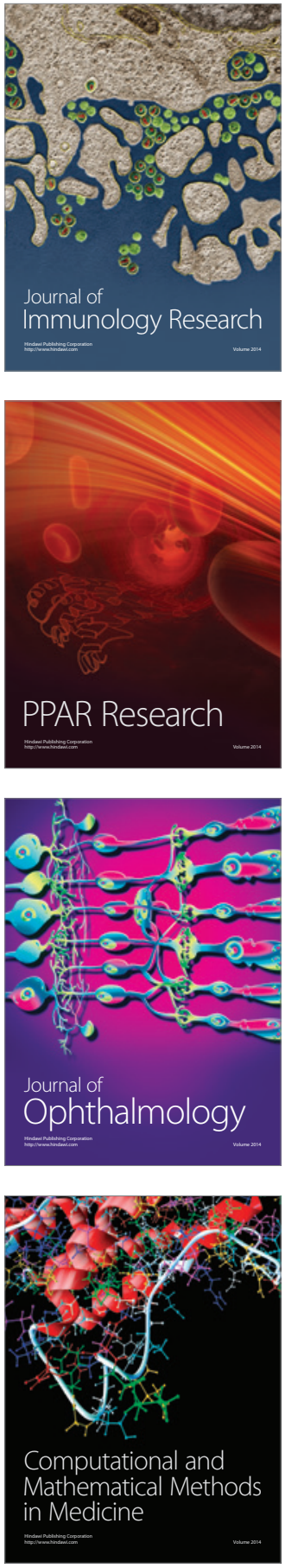

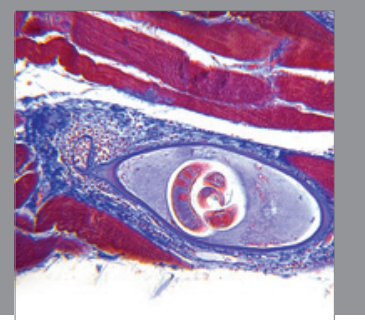

Gastroenterology

Research and Practice
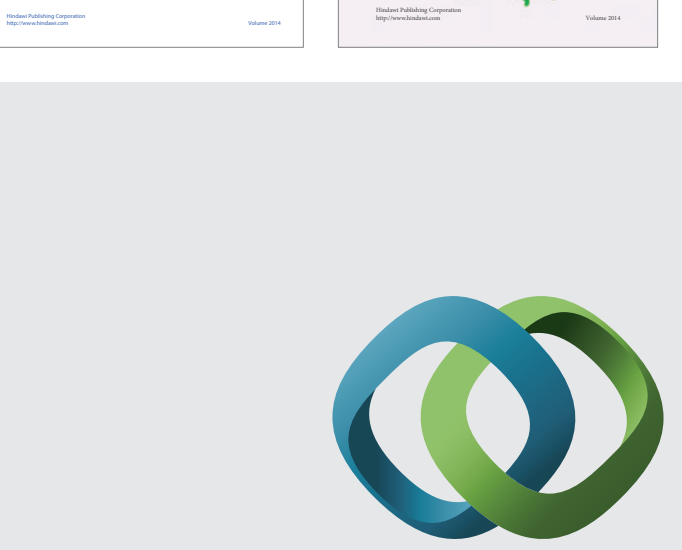

\section{Hindawi}

Submit your manuscripts at

http://www.hindawi.com
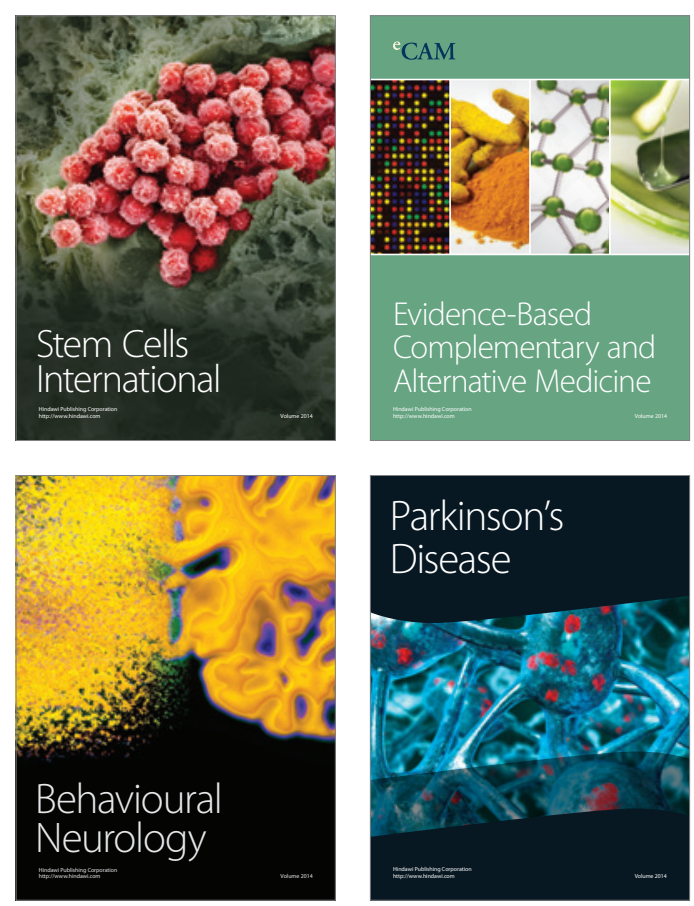

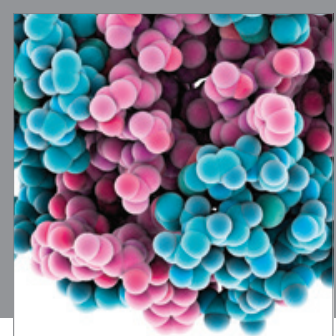

Journal of
Diabetes Research

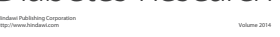

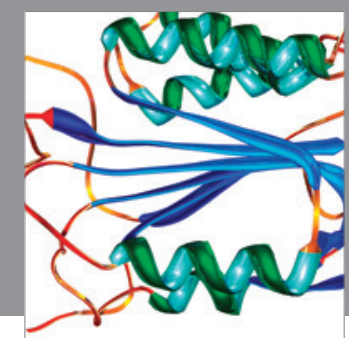

Disease Markers
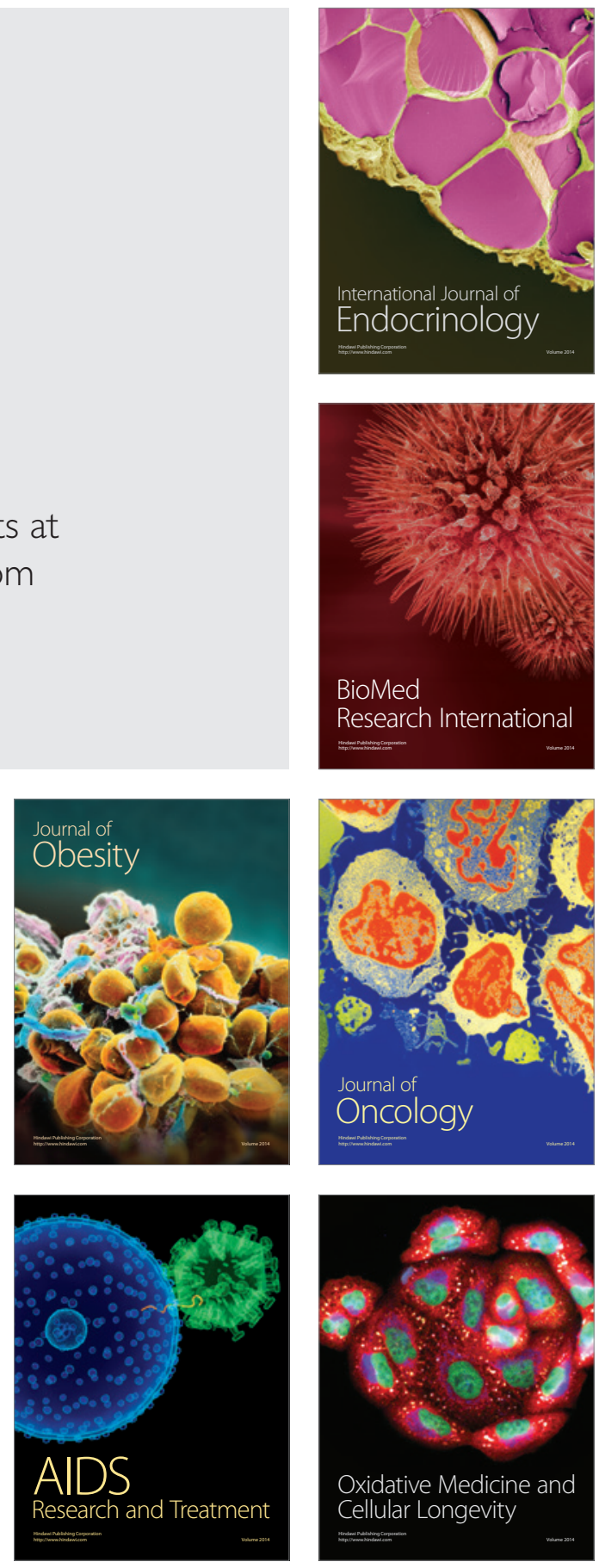